\title{
Clostridium septicum
}

National Cancer Institute

\section{Source}

National Cancer Institute. Clostridium septicum. NCI Thesaurus. Code C86292.

A species of anaerobic, Gram positive, rod shaped bacteria assigned to the phylum

Firmicutes. This species is sporeforming, hemolytic, produces exotoxins and is

lecithinase, lipase, indole and urease negative. C. septicum is highly pathogenic; infection

is most commonly associated with paraneoplastic syndrome and it is also a causative agent of gas gangrene. 